\title{
Noninvasive Follicular Thyroid Neoplasm with Papillary-Like Nuclear Features: From Echography to Genetic Profile
}

\author{
Francesca Maletta, ${ }^{1}$ Enrico Costantino Falco, ${ }^{2}$ Alessandro Gambella, ${ }^{2}$ \\ Jasna Metovic ${ }^{3}$ and Mauro Papotti ${ }^{3}$ \\ ${ }^{1}$ Pathology Unit, Department of Laboratory Medicine, AOU Città della Salute e della Scienza di Torino, Turin, \\ Italy \\ ${ }^{2}$ Pathology Unit, Department of Medical Sciences, University of Turin, Turin, Italy \\ ${ }^{3}$ Pathology Unit, Department of Oncology, University of Turin, Turin, Italy
}

\begin{abstract}
In thyroid pathology, the great variety of types and the wide range of aggressiveness of thyroid cancers complicate both diagnosis and management. In 2016, a subset of noninvasive encapsulated follicular variant of papillary thyroid carcinoma was reclassified as noninvasive follicular thyroid tumor with papillarylike nuclear features (NIFTP) to reduce overtreatment of this low-risk tumor that follows a benign course after surgery. Starting from a paradigmatic clinical case, in this short review, we will summarize the ultrasonography, cytological, histological and molecular features of this new entity. In the preoperative settings, the recognition of some peculiar elements may only suggest the possibility of a NIFTP, thus favoring a less aggressive surgical approach. However, the diagnosis of NIFTP can only be made after complete resection of the lesion by detecting well-defined inclusion and exclusion histopathological criteria. Since NIFTP is not 'malignant,' surgery may be considered curative with no further treatment or surveillance needed. NIFTP-related issues, including nodule size, multifocality, oncocytic changes, heterogeneous incidence across different geographical areas and its occurrence in the pediatric age, will be discussed.
\end{abstract}

Keywords: borderline tumors; cytology; noninvasive follicular thyroid neoplasm with papillary-like nuclear features; thyroid; ultrasound

Tohoku J. Exp. Med., 2020 November, 252 (3), 209-218.

\section{Introduction}

In thyroid pathology, the great variety of types and the wide range of aggressiveness of thyroid cancers complicate both diagnosis and management. In fact, thyroid carcinomas are a heterogeneous group of neoplasms which behave in extremely different ways depending on the histotype, ranging from the "nearly normal" life expectancy of most patients with papillary carcinoma (PTC) to the few months survival in patients affected by anaplastic carcinoma (Meissner and Warren 1969; Lloyd et al. 2017). In past years, lack of consensus among experts in endocrine/thyroid pathology raised questions about the proper classification of these tumors, with subsequent difficulties in patients' management. However, efforts have been made over the years in order to deliver uniform diagnoses as much as possible.
For example, in cytology, the introduction of the Bethesda System for Reporting Thyroid Cytology (TBSRTC) in 2009 (Cibas and Ali 2009) offered a standardized thyroid fine-needle aspiration biopsy terminology with six diagnostic categories, with an implied risk of malignancy and management recommendation for each of them: this determined a positive impact on cytological diagnosis and clinical management.

Another example of this "struggle for uniformity" was offered by the "Turin proposal" in 2007: an international group of expert thyroid pathologists met in Turin (Italy) to define the diagnostic morphological criteria of a distinct group of thyroid carcinomas with a behavior intermediate between well-differentiated (papillary and follicular) and undifferentiated (anaplastic) carcinomas: they defined these tumors as "poorly differentiated thyroid carcinomas"

Received July 30, 2020; revised and accepted October 2, 2020. Published online October 22, 2020; doi: 10.1620/tjem.252.209.

Correspondence: Francesca Maletta, M.D., P Pathology Unit, Department of Laboratory Medicine, AOU Città della Salute e della

Scienza di Torino, Via Santena 7, Turin 10127, Italy.

e-mail: francesca.maletta@libero.it

(C)2020 Tohoku University Medical Press. This is an open-access article distributed under the terms of the Creative Commons Attribution-NonCommercial-NoDerivatives 4.0 International License (CC-BY-NC-ND 4.0). Anyone may download, reuse, copy, reprint, or distribute the article without modifications or adaptations for non-profit purposes if they cite the original authors and source properly.

https://creativecommons.org/licenses/by-nc-nd/4.0/ 
(Volante et al. 2007), thus unifying under a single term and with strict morphological criteria what in previous years had been defined with heterogeneous and confounding approaches and terminology (insular, primordial cell, trabecular carcinoma, and others).

One of the most recent and relevant changes in the classification of thyroid tumors, however, is in the field of the so-called "neoplasms with indolent behavior." It is well known that one of the most serious problems in thyroid pathology is the risk of overdiagnosis and overtreatment for low-risk patients with indolent disease: on the one side, in fact, a more vigilant clinical surveillance has determined a rising incidence in the detection of subcentimeter, incidental carcinomas; on the other, the increasing recognition of certain variants, most notably the follicular variant of papillary thyroid carcinoma (FVPTC), which, if encapsulated and not invasive, has an indolent behavior despite the designation as "carcinoma", can contribute to the problem of overdiagnosis and overtreatment.

Recently, an international multidisciplinary collaborative group headed by Dr. Nikiforov (Nikiforov et al. 2016) established the criteria to reclassify a specific subgroup of encapsulated/noninvasive follicular variant of papillary thyroid carcinoma as "noninvasive follicular thyroid neoplasm with papillary-like nuclear features" (NIFTP), in order to downgrade these very-low risk neoplasms and thus reduce the risk of overdiagnosis and overtreatment.

Starting from a paradigmatic clinical case, the goal of this review is to provide a brief summary on the new entity termed NIFTP focusing on all its features, from ultrasound and cytological features to post-surgery histological diagnosis and genetic profile.

\section{Clinical Case}

A single $25-\mathrm{mm}$ nodule in the left thyroid lobe was incidentally discovered in a 55-year-old woman during doppler ultrasonographic evaluation of cervical vessels. The ultrasound pattern revealed a hypoechoic nodule, with rounded and well-defined margins, intra and perinodal vascularization and an intermediate elastosonographic pattern: the risk category according to the 2016 AACE/ACE/AME US classification system was intermediate (Gharib et al. 2016). The nodule was submitted to fine needle aspiration biopsy (FNA). Both alcohol-fixed Hematoxylin and Eosin or Papanicolaou-stained smears and paraffin-embedded cell-block sections revealed a highly cellular microfollicular patterned lesion, with scant colloid (Fig. 1). The nuclei were at least twice the size of a red blood cell and showed focal clearing and some irregularities of nuclear contour (grooves, indentations). No pseudoinclusions were observed. A diagnosis of follicular neoplasm (FN) (Category IV sec. Bethesda) (Cibas and Ali 2017) was rendered and, in agreement with the standards of care in Italy, one month later the patient underwent left hemithyroidectomy. Gross sampling confirmed the presence of a single, $25 \mathrm{~mm}$ nodule, which was entirely sampled. Histologically, the nodule was surrounded by a thin and complete capsule and had a microfollicular architecture, with scattered macrofollicles. No papillary formations were observed. The thyrocytes showed large nuclei, nearly twice the diameter of the nuclei of the surrounding normal thyroid parenchyma, with clearing and irregularities of the nuclear membrane contour (grooves) (Fig. 1). Colloid was dense. No sign of vascular or capsular invasion was present. Thus, a diagnosis of noninvasive follicular thyroid neoplasm with papillary-like nuclear features" (NIFTP) was made. No completion thyroidectomy was performed and radioiodine was not administered. The patient is alive and well two years after diagnosis.

\section{NIFTP in the Pre-Operative Setting}

When Nikiforov and coworkers (2016) proposed the term NIFTP, it was already known that encapsulated/welldemarcated forms of FVPTC without vascular/capsular invasion had an excellent prognosis after complete resection, even if treated with more conservative approaches (lobectomy rather than total thyroidectomy) (Liu et al. 2006; Rivera et al. 2010; Perros et al. 2014; Rosario et al. 2014; Ganly et al. 2015). In particular, some Authors (Kakudo et al. 2012) suggested that encapsulated forms of FVPTC should be classified into a unified category of "borderline malignancy" together with other lesions, including follicular adenoma with PTC-like nuclei, non-encapsulated tumors (papillary microcarcinoma) and encapsulated tumors: these latter include the follicular carcinoma with capsular invasion only (minimally invasive follicular carcinoma) and the so-called tumors with uncertain malignant potential (UMP), that is the Well-Differentiated Tumor of UMP (WDT-UMP, with questionable nuclear features, suspicious but not diagnostic of PTC-type nuclei) and Follicular Tumor of UMP (FT-UMP, with regular nuclei but questionable capsular or vascular invasion) (Williams 2000; Kakudo 2018). In particular, WDT-UMP have morphological, immunohistochemical and molecular characteristics distinct from PTC and a general favorable outcome, thus favoring a definition of WDT with uncertain behavior (WDT-UB) according to Liu et al. (2011). Recently, Ohba et al. (2019) describe a novel borderline tumor with a papillary structure and delicate nuclear changes which might turn into a possible novel subtype of borderline tumor: the Authors defined it "noninvasive encapsulated papillary RAS-like thyroid tumor (NEPRAS)" and eliminate the word "cancer" in a way similar to the definition of NIFTP. A unified nomenclature for these lesions was hypothesized to be helpful to reduce the significant observer disagreements in the pathological diagnosis of this problematic group of tumors.

Deep differences exist in the management of thyroid nodules among different geographical areas (Kakudo 2020) and the reasons for these differences will be discussed in the following paragraphs; however in Western countries hemithyroidectomy is nowadays widely accepted as the 

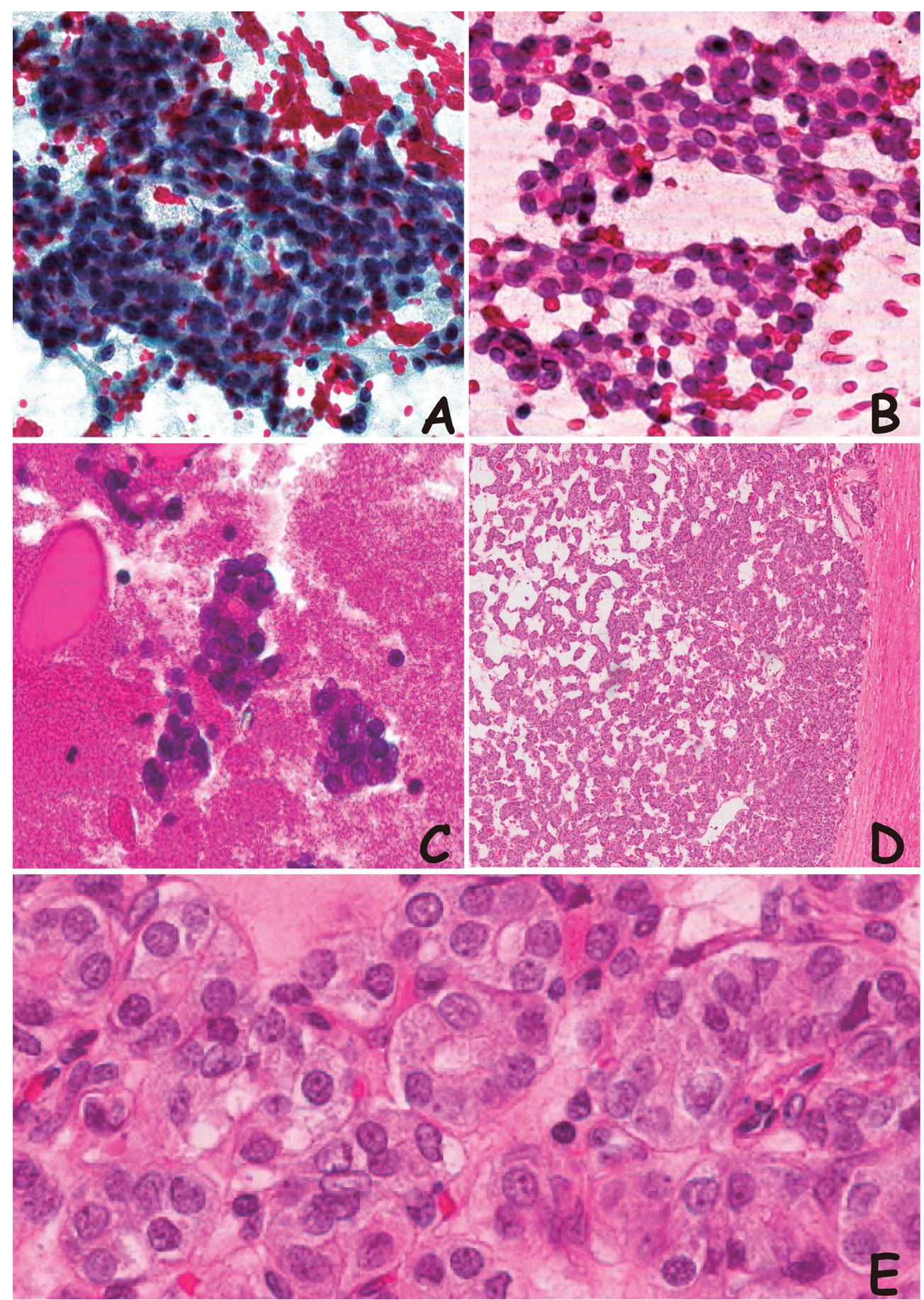

Fig. 1. Cytological and histological appearance of a $25-\mathrm{mm}$ thyroid nodule.

In FNA (A, B, smears stained with Papanicolaou and H\&E, $400 \times$ magnification; and C, cell-block section stained with $\mathrm{H} \& \mathrm{E}, 400 \times$ magnification), the nodule shows microfollicles with nuclear enlargement (defined as nuclei being at least twice the size of a red blood cell), irregularities of contour, with focal nuclear clearing; a diagnosis of Bethesda Category IV was rendered. At histology, the same lesion appears well capsulated and shows a predominant microfollicular architecture $(\mathrm{D} ; \mathrm{H} \& \mathrm{E}$ stain, $100 \times$ magnification). At high magnification $(\mathrm{E} ; \mathrm{H} \& \mathrm{E}, 400 \times$ magnification) the nuclei are clear, large and show irregularities of nuclear contour and grooves. A diagnosis of NIFTP was thus formulated.

FNA, fine needle aspiration; H\&E, hematoxylin and eosin.

standard of care for patients with NIFTP and for this reason, a preoperative recognition or at least a suspicion of NIFTP would be useful in guiding subsequent therapeutic decisions, avoiding unnecessary overtreatment (total thy- roidectomy and/or lymphadenectomy). However, definitive criteria for recognizing potential NIFTP in the preoperative setting have not been established to date, and, at present, NIFTP can only be suspected before surgery: as it will be 
discussed later, the definitive diagnosis in fact relies on histological examination of the resected nodule.

However, efforts have been made in order to find some ultrasonographical, cytological (Rosario 2017; Strickland et al. 2018; Bongiovanni et al. 2019b) and molecular features (Nikiforov 2017) that can be useful for suspecting NIFTP in the pre-operative setting.

\section{Ultrasound}

NIFTP nodules tend to be circumscribed, oval/round in shape, iso or hypoechoic with a hypoechoic rim, and usually with an intranodular vascular flow at the Color flow Doppler. They have no signs of extrathyroidal extension, microcalcification, taller-than-wide shape or spiculate/ microlobulate/ill-defined margin, and show absence of regional lymph node involvement. These same features are usually shared with the minimally invasive encapsulated follicular variant of papillary thyroid carcinoma (EFVPTC), while the overtly invasive EFVPTC typically shows a round/oval hypoechoic nodule with irregular margins. Conversely, infiltrative FVPTC tend to be markedly hypoechoic, taller-than-wide, with microcalcifications and/ or blurred margins and mostly avascular on Color flow Doppler (Yang et al. 2017; Rosario and Mourão 2019). Multiple ultrasound scores are used to classify thyroid nodules and guide the subsequent diagnostic-therapeutic strategy. Based on the above listed ultrasound characteristics, NIFTP are usually classified as low or intermediate risk lesions. Therefore, these US scores are able to separate NIFTP from most infiltrative FVPTC, but only from a few EFVPTC (Yang et al. 2020).

\section{Cytopathology}

Retrospective studies on fine-needle aspiration biopsies have shown that the cytological diagnoses associated with NIFTP include a wide spectrum of findings and can potentially belong to any of the six categories of TBSRTC; however, the majority of cases of NIFTP fall into one of the three so-called "indeterminate" categories: 1) atypia of undetermined significance/follicular lesion of undetermined significance (AUS or category III); 2) suspicious for follicular neoplasm/follicular neoplasm (SFN or category IV); and 3) suspicious for malignancy (SUS or category V) (Maletta et al. 2016; Strickland et al. 2018). Strickland et al (2018) suggested that a limited set of cytological features is associated with a likely histological diagnosis of NIFTP: a predominantly microfollicular growth pattern and the presence of nuclear pallor, distinct nucleoli, and irregular nuclear membrane contours are useful criteria to distinguish potential NIFTP from benign nodules.

The redefinition of NIFTP as a neoplasm rather than a carcinoma is expected to alter the intrinsic risk of malignancy for the diagnostic categories of TBSRTC. Several papers dealt with this topic and they all report that the downgrading of NIFTP in the "not malignant"category has an impact mainly on the indeterminate categories (category
III-IV-V of Bethesda System), where the risk is expected to be reduced, while the benign (II) and the malignant categories (VI) are less affected (Maletta et al. 2017). In the study by Linhares and coworkers (2020), the risk was reduced by $7 \%, 15 \%$, and $2 \%$ in category III, IV and V respectively, while a meta-analysis by Bongiovanni and coworkers (2019a) showed that the introduction of NIFTP led to a reduction of the risk of malignancy for the TBSRTC diagnostic categories V and VI by 14 and 3\%, respectively. Clinicians should be aware of these data to avoid overtreatment: the new TBSRTC (Cibas and Ali 2017) contains some comments and notes about this shift in order to help guiding surgical management. The Bethesda system is the most popular diagnostic system for thyroid cytology in Asian countries, too; however, in this continent, a less aggressive clinical management is recommended for patients with indeterminate thyroid nodules (categories III and IV) (Kakudo et al. 2017). In Western countries, clinical guidelines (Haugen et al. 2016; Ferris et al. 2018) usually recommend surgery for these patients (or to perform molecular tests, such as gene-expression classifiers which, however are usually not available outside of North America) (Kakudo 2020), because malignancy cannot be completely ruled out without histology, and this is what happened to the 55-year-old patient of the clinical case above mentioned. Conversely, in Asia indeterminate thyroid nodules with no suspicious clinical findings usually undergo surveillance and diagnostic surgery is not performed for these indeterminate nodules: this results in low rates of borderline tumors in surgically-treated patients and in reduction of overtreatment of indolent thyroid tumors; another consequence is that a higher "risk of malignancy" for indeterminate cytological categories is achieved in Asian practices, though using the same Bethesda system.

\section{Genetic profile}

Almost $80 \%$ of NIFTP demonstrate clonal alterations. As predicted, NIFTP molecular profile is similar to that of EFVPTC and of follicular adenoma and carcinoma, with a high prevalence of RAS and absence of BRAF V600E mutations or RET/PTC rearrangements, which are more commonly associated with non-encapsulated FVPTC or classical PTC (Nikiforov et al. 2016; Bizzarro et al. 2016). Based on this molecular profile, NIFTP could be a precursor tumor of EFVPTC, a prevalently indolent carcinoma with low-malignant potential, but not of infiltrative FVPTC, which is associated to a worse prognosis and occasional lethal outcome (Rivera et al. 2010). Other mutations/ fusions documented also include: PAX8/PPARG and THADA fusions, as well as EIF1AX and BRAF K601E mutations (Seethala et al. 2018). In cytology specimens, molecular testing has its utility mainly in the study of thyroid nodules with a diagnosis that falls in one of the indeterminate categories. Paulson et al. (2017) demonstrated that over half $(59 \%)$ of a past series of tumors with RAS mutations would now be diagnosed as NIFTP and these are 
typically associated with an indeterminate diagnosis on FNA material. Other molecular approaches helpful for the triage of indeterminate thyroid nodules include the use of miRNA expression profiles (Borrelli et al. 2017) or gene expression classifiers (Jiang et al. 2016).

\section{Histology of NIFTP}

As stated above, the diagnosis of NIFTP can only be made on histological samples after complete resection. The diagnostic criteria listed in the first report introducing NIFTP (Nikiforov et al. 2016) and in the following revisions (Lloyd et al. 2017; Nikiforov et al. 2018; Seethala et al. 2018) include (i) the nodule has to be encapsulated or, if unencapsulated, has to be well demarcated from the adjacent parenchyma, showing a discrete interface with expansive growth; (ii) vascular or capsular invasion are absent after thorough microscopic capsular examination; (iii) architecture is follicular (micro or macrofollicular or a mixture of both), with no well-formed papillae, no psammoma bodies and $<30 \%$ solid/trabecular/insular growth pattern; (iv) nuclei of thyrocytes forming the lesion share the nuclear features of PTC (large, clear nuclei with irregularities of nuclear contour): however, NIFTP nuclear features tend to be typically more subtle, and range from diffuse in distribution to patchy and multifocal; (v) no tumor necrosis nor high mitotic activity are present (Figs. 2, 3).

Molecular tests are helpful but not required for NIFTP diagnosis. BRAFV600E, RET/PTC fusions or gene mutations such as TERT promoter or TP53 exclude a diagnosis of NIFTP (Nikiforov et al. 2018), but it has been observed that if the morphological diagnostic criteria are strictly observed, these mutations are very unlikely to occur (Nikiforov et al. 2016; Johnson and Sadow 2018; Jung et al. 2018; Kim et al. 2018a), apart from some exceptional cases (Kim et al. 2018b).

The main differential diagnoses of NIFTP include: follicular adenoma, from which NIFTP differs because of the presence of PTC-like nuclei; encapsulated follicular variant of PTC, which shows focal or extensive capsular and/or vascular invasion; infiltrative follicular variant of PTC which has no capsule or demarcation, but rather shows an infiltrative pattern of growth into the surrounding thyroid parenchyma; other borderline capsulated and follicular-pattern tumors such as WDT-UMP, FT-UMP (follicular tumor of uncertain malignant potential), WDT-UB (well-differentiated tumor with uncertain behavior); and the recently introduced borderline tumor with papillary structure "NEPRAS," mentioned above (Ohba et al. 2019).

If encountering a lesion which might be diagnosed as NIFTP, it is mandatory for pathologists to follow some rules (Seethala et al. 2018): during grossing, the entire tumor capsule or tumor-normal interface should be submitted for histologic evaluation; multiple sections can be submitted per block, focusing on the tumor periphery and its junction to the parenchyma.

Since NIFTP is not a carcinoma, it does not require a

\section{Diagnostic criteria of NIFTP}

A) Margins

Expansive Infiltrative

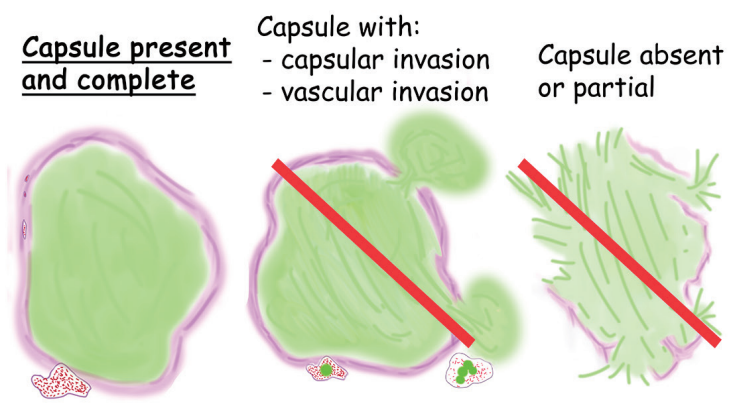

B) Architecture

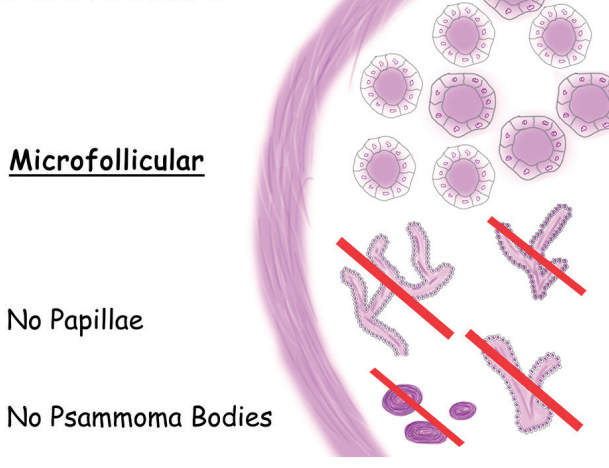

C) Cytology
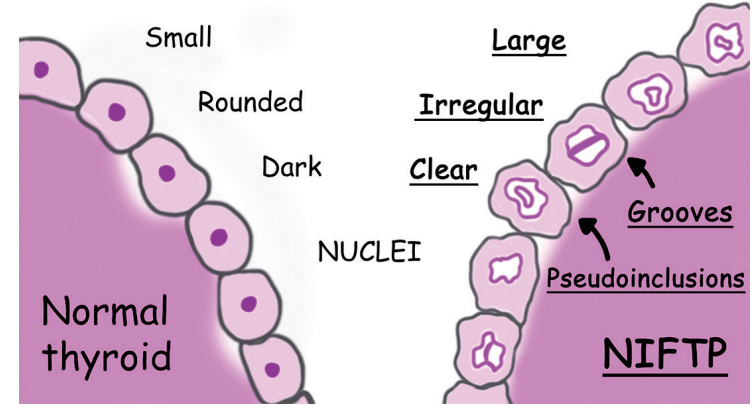

Fig. 2. Main morphological features of NIFTP.

A) Margins of the nodule: a NIFTP has definite, expansive margins, usually encircled by a continuous, intact capsule (left). If capsular or vascular invasions are present (middle), or if the capsule is discontinuous or completely absent and the nodule has infiltrative margins towards the surrounding parenchyma (right), then the lesion cannot be diagnosed as NIFTP.

B) Architecture of the nodule: a NIFTP as a prevalent microfollicular pattern of growth (up) and no papillae or psammoma bodies are allowed (down).

C) Nuclear features: NIFTP have large, clear and irregular nuclei, with frequent grooves and occasional pseudoinclusions. NIFTP nuclei (right) differ significantly from regular nuclei of normal thyrocytes (left), which are small, rounded and dark. 


\section{DIAGNOSTIC FEATURES OF NIFTP}
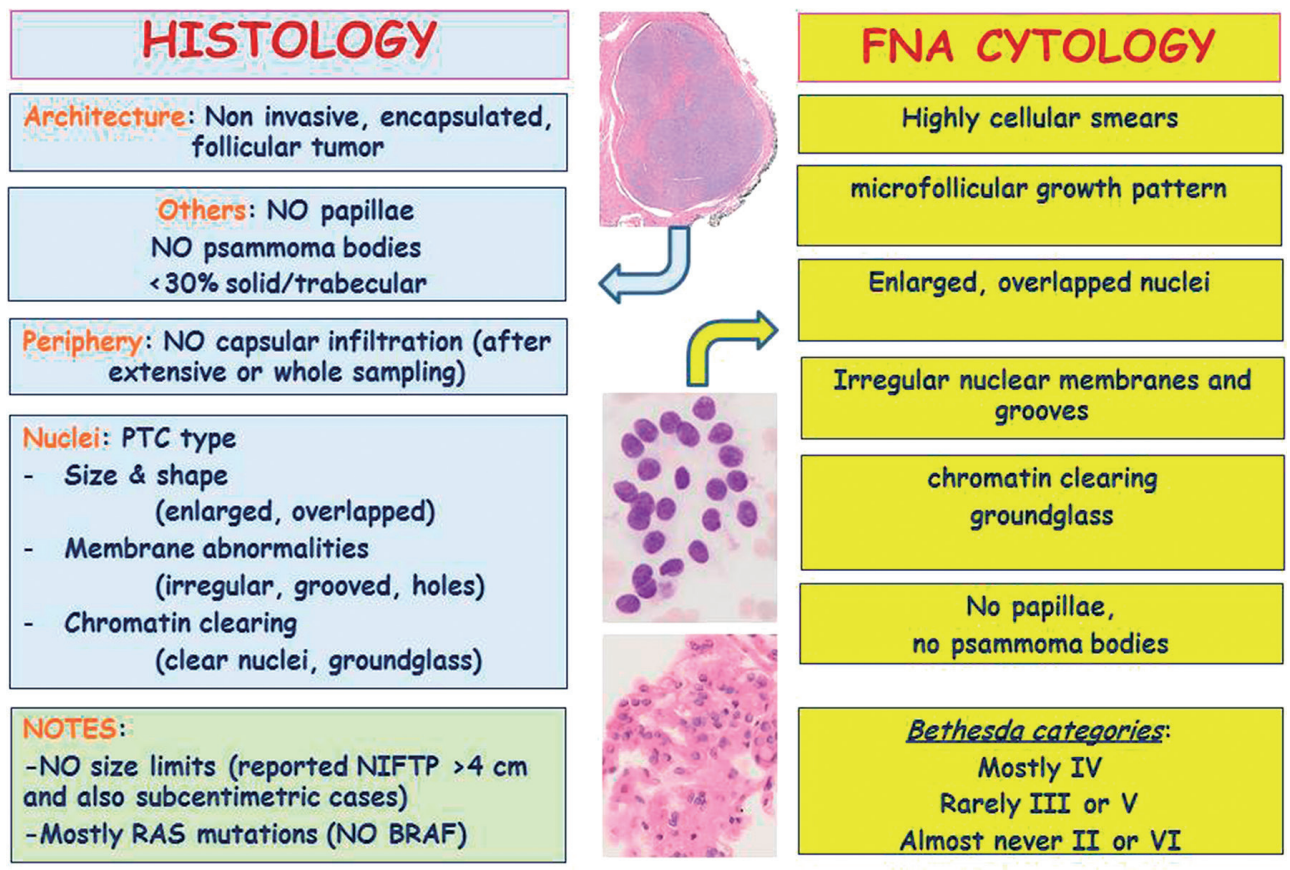

Fig. 3. Summary of histological and cytological features of NIFTP.

The figure summarizes the histological (left) and cytological (right) diagnostic features of NIFTP.

formal TNM staging, but a limited data set consisting of tumor size, laterality and margin status may be useful.

\section{Open Issues on NIFTP}

\section{Can a NIFTP be of large size?}

Most NIFTP are between 2 and $3 \mathrm{~cm}$, but the size of the tumor does not represent a criterion of exclusion for the diagnosis of NIFTP (Nikiforov et al. 2018). Large NIFTP (defined as $>4 \mathrm{~cm}$ ) require greater care and time-consuming evaluation, with extensive sampling in order to exclude invasion, the presence of papillae or other excluding findings. However, different studies showed that, if all diagnostic criteria are carefully checked and met, even large NIFTP are indolent after a long term follow up (Xu et al. 2017; Chu and Sadow 2020). Therefore, no upper dimensional limit exists for the diagnosis of NIFTP.

\section{Can a NIFTP be of small size?}

In the original article defining NIFTP (Nikiforov et al. 2016), all the cases were $1 \mathrm{~cm}$ or greater in size and none was in the "microcarcinoma size" $(\leq 1 \mathrm{~cm})$ range. However, subsequent studies revealed that also subcentimeter lesions meeting the histologic criteria of NIFTP behave in a very low-risk manner and thus deserve to be diagnosed as micro-NIFTP (Xu et al. 2018; Shafique et al. 2018; LiVolsi and Baloch 2020).

\section{Can a NIFTP be multifocal?}

Tumor multifocality may be seen with any thyroid gland tumor. In the same thyroid, two or more NIFTP may coexist and this occurrence apparently does not exclude the diagnosis, however the issue of multifocal NIFTP needs to be further investigated (LiVolsi and Baloch 2020). In other cases, NIFTP may coexist with a carcinoma. A recent study by Zajkowska et al (2020) revealed that a synchronous malignant lesion could be present in a not negligible percentage of patients with NIFTP (15-46\%, mostly papillary thyroid microcarcinomas): if this happens, NIFTP should not be included in TNM staging, as in general, only malignant tumors should be incorporated into the staging systems (Seethala et al. 2018).

\section{Can a NIFTP be oncocytic?}

The original study cohort of NIFTP by Nikiforov and coworkers (2016) deliberately excluded tumors with oncocytic features, therefore such lesions were still labeled as FVPTC, oncocytic variant. However, a more recent study (Xu et al. 2019) on 61 patients bearing an oncocytic noninvasive EFVPTC demonstrated that these cases behaved like NIFTP, with no distant or lymph node metastasis or local recurrence in the entire cohort. Moreover, "oncocytic NIFTP" share the same RAS mutations as conventional NIFTP, though associated to a higher frequency of mitochondrial DNA mutations and multiple chromosomal gains/ losses. Therefore, even if awaiting further confirmatory studies, evidence exist that oncocytic not invasive EFVPTC should be renamed as "oncocytic NIFTP", in order to avoid overtreatment of these similarly indolent tumors (Xu et al. 
2019).

What is the incidence of NIFTP in different geographic areas?

The prevalence of NIFTP in different geographic areas is highly heterogeneous. In particular, the incidence of NIFTP is reported to be significantly lower in Asian Countries (1.6\%) compared to Western Countries (13.3\%) (Bychkov et al. 2018a; Hirokawa et al. 2020). Possible explanations of this difference may be a peculiar genetic profile of the lesions based on the ethnic background and the iodine intake or the different perception of histological diagnostic thresholds. However, some authors attribute part of this difference to other factors: the fear of medical malpractice claims, in particular, is higher in Western countries (especially North America) than in Asian countries (Kakudo 2020); the use of defensive medicine leads to higher rates of diagnostic surgery and to lower "diagnostic threshold for malignancy", increasing the number of lesions defined as borderline or malignant (Renshaw and Gould 2002; Labarge et al. 2018). A recent study by Hirokawa and coworkers (2020) showed that in a Japanese retrospective case series, the main differential diagnosis of NIFTP is follicular adenoma rather than PTC (probably because of a higher threshold for identifying the PTC nuclei by Eastern pathologists). However, since the clinical management of follicular adenoma and NIFTP is the same, there is no real utility in distinguishing between these two entities. Differences also concern the management of these borderline lesions. As already mentioned, Western guidelines recommend hemithyroidectomy for TBSRTC category IV nodules at FNA, considering the estimated risk of malignancy too high for observation. Conversely, in the Asian practice, active surveillance and strict triage for surgery is the standard of care for borderline lesions and low-risk carcinomas (Kakudo et al. 2017; Kakudo 2020). Moreover, the active surveillance for indeterminate nodules promoted by Japanese Institutions establishes a new paradigm to reduce overtreatment of these patients: since NIFTP is largely represented in the indeterminate cytological categories and for these categories the active surveillance is preferred to surgery, NIFTP in the Asian series tends to have low prevalence, with a subsequent low impact on the risk of malignancy in cytopathology (Bychkov et al. 2018b; Kakudo et al. 2018). As a conclusion, in the Asian background, the NIFTP category turns out to be less used and less useful, and its introduction seems to have a lower impact on the clinical practice (Seo et al. 2019; Hirokawa et al. 2020).

\section{Can NIFTP occur in children?}

In the pediatric population, NIFTP seems to be less frequent, probably due to the higher prevalence of classical PTC and other aggressive variants. As for the adult population, pediatric NIFTPs usually show a low-intermediate risk at the ultrasound evaluation and at FNA are classified within the indeterminate categories (Bethesda III, IV and V) (Rosario and Mourão 2018). Since pediatric PTCs usually have clinicopathological and molecular features that differ from those of adults and tend to be more aggressive (with higher recurrence rates), many authors raise doubts and concern as to whether NIFTP in children should be treated in the same way as adults. However, the few reported NIFTP cases in the pediatric population showed an indolent behavior, with no lymph node or distant metastases or local recurrences (Rossi et al. 2018; Rosario and Mourão 2018; Wang et al. 2020). The current data therefore suggest that even pediatric NIFTP can be managed in a more conservative way, with lobectomy and no lymph node dissection or subsequent radioablation therapy.

\section{Management after Surgery}

It is expected that specific protocols or recommendations for selective follow-up of subgroups of patients with NIFTP will be incorporated in future guidelines, but at present, the debate on management of patients with completely resected NIFTP is still ongoing (Haugen et al. 2017): some authors recommend NIFTP to be monitored in the same way as low-risk PTC (Cho et al. 2017; Parente et al. 2018). Others think that if histological criteria are strictly applied, this approach seems too cautious and unnecessary and believe that NIFTP with no other associated malignancy, nor other nodules in the contralateral lobe (detected by ultrasounds, if lobectomy only was performed) should be managed like a follicular adenoma. In this respect, Rosario and coworkers (2018) noticed that if NIFTP patients were submitted to a post-surgery follow-up similar to that of lowrisk PTC patients, the costs of detecting a potential very rare recurrence would be too high, requiring hundreds of laboratory and ultrasound tests along the required 10 years of follow-up, with the risks of false-positive results and their potential consequences.

\section{Conclusion}

In recent decades, the rising incidence of PTC could be at least in part attributed to the inclusion of noninvasive encapsulated EFVPTC in the malignant category. This variant accounts for $10-20 \%$ of all thyroid malignancies (Lloyd et al. 2017) but, despite its designation as carcinoma, it appears to follow an indolent course, with an excellent prognosis. Moreover, several authors reported that the diagnosis of noninvasive EFVPTC is afflicted by high rate of subjectivity in pathological interpretation, since it relies solely on the detection of proper papillary-like nuclear irregularities (which are only partially or focally expressed in this variant) and not on other more established and reproducible criteria, such as capsular or vascular invasion (Maletta et al. 2017). As a consequence, the differential diagnosis between EFVPTC and benign entities, such as microfollicular goiter or follicular adenoma, can be challenging, and attempts have been made to solve the problem, introducing intermediate categories such as "atypical ade- 
noma" or "well-differentiated tumors of uncertain malignant potential" (Williams 2000; Lloyd et al. 2017). Nonetheless, until recent years, patients receiving a diagnosis of EFVPTC without invasion continued to be treated with therapies similar to those of the more aggressive papillary or follicular thyroid carcinoma.

Noninvasive follicular thyroid neoplasm with papillary-like nuclear features (NIFTP) is a new terminology that applies to encapsulated or clearly delimited, noninvasive neoplasm with a follicular growth pattern and nuclear features of papillary thyroid carcinoma (Nikiforov et al. 2016, 2018; Lloyd et al. 2017): it identifies a substantial category of "borderline" neoplasms (Lloyd et al. 2017; Rosario and Mourão 2019), previously defined malignant. Its introduction as a new diagnostic entity had a strong impact in Western countries, because it downgraded former "not invasive EFVPTC" from the "carcinoma category" to the "borderline category", thus reducing overtreatment. As already stated, the surgical approach for indeterminate nodules is typical of Western countries, while in Asia other approaches prevail. That is why the impact of NIFTP was less revolutionary in Asian world, where indolent, low-risk neoplasms usually undergo surveillance rather than surgery and overtreatment is a minor problem. Kakudo (2018) proposed to group together these low risk tumors into a single borderline tumors category, including encapsulated tumors (follicular carcinoma with capsular invasion only, encapsulated papillary carcinoma without invasion, WDT-UMP and FT-UMP) and non-encapsulated tumors (papillary microcarcinoma). These borderline/precursor thyroid tumors are indolent and should be treated more conservatively than was previously recommended by Western clinical guidelines for thyroid follicular cell carcinomas (total thyroidectomy, followed by radio-active iodine treatment).

In the pre-surgical phase, NIFTP cannot be recognized with certainty but only suspected: in the appropriate clinical context, that is where clinical and sonographic data support a low-risk lesion, and if cytological (and possibly molecular) findings are consistent with NIFTP, a lobectomy might be preferred to radical thyroidectomy as the initial surgical approach.

According to Western standards of practice, surgery is necessary for patients with NIFTP for two reasons: 1) the definitive diagnosis of NIFTP relies on histological criteria to exclude an invasive/infiltrative FVPTC or other type of thyroid carcinoma and NIFTP cannot be a cytological diagnosis (NIFTP mainly falls in Bethesda cytological categories III, IV and V, which have a ROM between 30\% and 83\%) (Bychkov et al. 2018b); and 2) some Authors hypothesized that NIFTP may represent a precursor lesion to thyroid malignancy and, should that be the case, surgery can be considered both diagnostic and curative (Nikiforov et al. 2016; Strickland et al. 2018). However, we believe that theories about the evolution of NIFTP are, at present, mere speculations: NIFTP is a histological diagnosis and reflects the features of the nodule at the time of surgery. No con- clusions can be drawn on what it would become if left untreated without surgery.

Clear-cut diagnostic histological criteria to define a NIFTP are essential (Fig. 3) to increase diagnostic reproducibility, while until the introduction of NIFTP, variations existed in the definition of EFVPTC, resulting in a significant interobserver disagreement in the final diagnosis of follicular thyroid neoplasms. Moreover, it prevents overtreatment and unnecessary anxiety in patients and reduces management costs of follow-up.

This proposal was endorsed by the World Health Organization (WHO) in the 4th edition of the classification of endocrine tumors (Lloyd et al. 2017). However, despite its inclusion, it may still take some time until the NIFTP nomenclature becomes routine in all pathology laboratories, especially if considering that relevant geographical differences exist in the incidence of NIFTP all over the world.

\section{Conflict of Interest}

The authors declare no conflict of interest.

\section{References}

Bizzarro, T., Martini, M., Capodimonti, S., Straccia, P., Lombardi, C.P., Pontecorvi, A., Larocca, L.M. \& Rossi, E.D. (2016) Young investigator challenge: the morphologic analysis of noninvasive follicular thyroid neoplasm with papillary-like nuclear features on liquid-based cytology: some insights into their identification. Cancer Cytopathol., 124, 699-710.

Bongiovanni, M., Faquin, W.C., Giovanella, L., Durante, C., Kopp, P. \& Trimboli, P. (2019a) Impact of non-invasive follicular thyroid neoplasms with papillary-like nuclear features (NIFTP) on risk of malignancy in patients undergoing lobectomy/thyroidectomy for suspected malignancy or malignant fine-needle aspiration cytology findings: a systematic review and meta-analysis. Eur. J. Endocrinol., 181, 389-396.

Bongiovanni, M., Giovanella, L., Romanelli, F. \& Trimboli, P. (2019b) Cytological diagnoses associated with noninvasive follicular thyroid neoplasms with papillary-like nuclear features according to the Bethesda System for Reporting Thyroid Cytopathology: a systematic review and meta-analysis. Thyroid, 29, 222-228.

Borrelli, N., Denaro, M., Ugolini, C., Poma, A.M., Miccoli, M., Vitti, P., Miccoli, P. \& Basolo, F. (2017) miRNA expression profiling of 'noninvasive follicular thyroid neoplasms with papillary-like nuclear features' compared with adenomas and infiltrative follicular variants of papillary thyroid carcinomas. Mod. Pathol., 30, 39-51.

Bychkov, A., Jung, C.K., Liu, Z. \& Kakudo, K. (2018a) Noninvasive follicular thyroid neoplasm with papillary-like nuclear features in Asian practice: perspectives for surgical pathology and cytopathology. Endocr. Pathol., 29, 276-288.

Bychkov, A., Keelawat, S., Agarwal, S., Jain, D., Jung, C.K., Hong, S., Lai, C.R., Satoh, S. \& Kakudo, K. (2018b) Impact of non-invasive follicular thyroid neoplasm with papillary-like nuclear features on the Bethesda system for reporting thyroid cytopathology: a multi-institutional study in five Asian countries. Pathology, 50, 411-417.

Cho, U., Mete, O., Kim, M.H., Bae, J.S. \& Jung, C.K. (2017) Molecular correlates and rate of lymph node metastasis of non-invasive follicular thyroid neoplasm with papillary-like nuclear features and invasive follicular variant papillary thyroid carcinoma: the impact of rigid criteria to distinguish non-invasive follicular thyroid neoplasm with papillary-like nuclear features. Mod. Pathol., 30, 810-825. 
Chu, Y.H. \& Sadow, P.M. (2020) Noninvasive follicular thyroid neoplasm with papillary-like nuclear features (NIFTP): diagnostic updates and molecular advances. Semin. Diagn. Pathol., 37, 213-218.

Cibas, E.S. \& Ali, S.Z. (2009) The Bethesda system for reporting thyroid cytopathology. Thyroid, 19, 1159-1165.

Cibas, E.S. \& Ali, S.Z. (2017) The 2017 Bethesda system for reporting thyroid cytopathology. Thyroid, 27, 1341-1346.

Ferris, R.L., Nikiforov, Y., Terris, D., Seethala, R.R., Ridge, J.A., Angelos, P., Duh, Q.Y., Wong, R., Sabra, M.M., Fagin, J.A., McIver, B., Bernet, V.J., Harrell, R.M., Busaidy, N., Cibas, E.S., et al. (2018) AHNS series: do you know your guidelines? AHNS endocrine section consensus statement: state-ofthe-art thyroid surgical recommendations in the era of noninvasive follicular thyroid neoplasm with papillary-like nuclear features. Head Neck, 40, 1881-1888.

Ganly, I., Wang, L., Tuttle, R.M., Katabi, N., Ceballos, G.A., Harach, H.R. \& Ghossein, R. (2015) Invasion rather than nuclear features correlates with outcome in encapsulated follicular tumors: further evidence for the reclassification of the encapsulated papillary thyroid carcinoma follicular variant. Hum. Pathol., 46, 657-664.

Gharib, H., Papini, E., Garber, J.R., Duick, D.S., Harrell, R.M., Hegedüs, L., Paschke, R., Valcavi, R. \& Vitti, P.; AACE/ACE/ AME Task Force on Thyroid Nodules (2016) American Association of Clinical Endocrinologists, American College of Endocrinology, and Associazione Medici Endocrinologi medical guidelines for clinical practice for the diagnosis and management of thyroid nodules: 2016 update. Endocr. Pract., 22, 622-639.

Haugen, B.R., Alexander, E.K., Bible, K.C., Doherty, G.M., Mandel, S.J., Nikiforov, Y.E., Pacini, F., Randolph, G.W., Sawka, A.M., Schlumberger, M., Schuff, K.G., Sherman, S.I., Sosa, J.A., Steward, D.L., Tuttle, R.M., et al. (2016) 2015 American Thyroid Association management guidelines for adult patients with thyroid nodules and differentiated thyroid cancer: the American Thyroid Association Guidelines task force on thyroid nodules and differentiated thyroid cancer. Thyroid, 26, 1-133.

Haugen, B.R., Sawka, A.M., Alexander, E.K., Bible, K.C., Caturegli, P., Doherty, G.M., Mandel, S.J., Morris, J.C., Nassar, A., Pacini, F., Schlumberger, M., Schuff, K., Sherman, S.I., Somerset, H., Sosa, J.A., et al. (2017) American Thyroid Association guidelines on the management of thyroid nodules and differentiated thyroid cancer task force review and recommendation on the proposed renaming of encapsulated follicular variant papillary thyroid carcinoma without invasion to noninvasive follicular thyroid neoplasm with papillary-like nuclear features. Thyroid, 27, 481-483.

Hirokawa, M., Higuchi, M., Suzuki, A., Hayashi, T., Kuma, S. \& Miyauchi, A. (2020) Prevalence and diagnostic significance of noninvasive follicular thyroid neoplasm with papillary-like nuclear features among tumors previously diagnosed as follicular adenoma: a single-institutional study in Japan. Endocr. J., doi: org/10.1507/endocrj.EJ20-0198. [Epub ahead of print].

Jiang, X.S., Harrison, G.P. \& Datto, M.B. (2016) Young investigator challenge: molecular testing in noninvasive follicular thyroid neoplasm with papillary-like nuclear features. Cancer Cytopathol., 124, 893-900.

Johnson, D.N. \& Sadow, P.M. (2018) Exploration of BRAFV600E as a diagnostic adjuvant in the non-invasive follicular thyroid neoplasm with papillary-like nuclear features (NIFTP). Hum. Pathol., 82, 32-38.

Jung, C.K., Kim, Y., Jeon, S., Jo, K., Lee, S. \& Bae, J.S. (2018) Clinical utility of EZH1 mutations in the diagnosis of follicular-patterned thyroid tumors. Hum. Pathol., 81, 9-17.

Kakudo, K. (2020) Asian and Western practice in thyroid pathology: similarities and differences. Gland Surg., doi:10.21037/gs-2019-catp-02. [Epub ahead of print].
Kakudo, K. (2018) How to handle borderline/precursor thyroid tumors in management of patients with thyroid nodules. Gland Surg.., 7, S8-S18.

Kakudo, K., Bai, Y., Liu, Z. \& Ozaki, T. (2012) Encapsulated papillary thyroid carcinoma, follicular variant: a misnomer. Pathol. Int., 62, 155-160.

Kakudo, K., Bychkov, A., Bai, Y., Li, Y., Liu, Z. \& Jung, C.K. (2018) The new 4th edition World Health Organization classification for thyroid tumors, Asian perspectives. Pathol. Int., 68, 641-664.

Kakudo, K., Higuchi, M., Hirokawa, M., Satoh, S., Jung, C.K. \& Bychkov, A. (2017) Thyroid FNA cytology in Asian practiceactive surveillance for indeterminate thyroid nodules reduces overtreatment of thyroid carcinomas. Cytopathology, 28, 455-466.

Kim, M.J., Won, J.K., Jung, K.C., Kim, J.H., Cho, S.W., Park, D.J. \& Park, Y.J. (2018a) Clinical characteristics of subtypes of follicular variant papillary thyroid carcinoma. Thyroid, 28, 311-318.

Kim, T.H., Lee, M., Kwon, A.Y., Choe, J.H., Kim, J.H., Kim, J.S., Hahn, S.Y., Shin, J.H., Chung, M.K., Son, Y.I., Ki, C.S., Yim, H.S., Kim, Y.L., Chung, J.H., Kim, S.W., et al. (2018b) Molecular genotyping of the non-invasive encapsulated follicular variant of papillary thyroid carcinoma. Histopathology, 72, 648-661.

Labarge, B., Walter, V., Lengerich, E.J., Crist, H., Karamchandani, D., Williams, N., Goldenberg, D., Bann, D.V. \& Warrick, J.I. (2018) Evidence of a positive association between malpractice climate and thyroid cancer incidence in the United States. PLoS One, 13, e0199862.

Linhares, S.M., Whitfield, B.W., Lee, A.F., Gordillo, D., Picado, O., Jeraq, M., Farrá, J.C. \& Lew, J.I. (2020) Impact of noninvasive follicular thyroid neoplasm with papillary-like nuclear features on revised bethesda system malignancy rates at a single institution. J. Surg. Res., 255, 152-157.

Liu, J., Singh, B., Tallini, G., Carlson, D.L., Katabi, N., Shaha, A., Tuttle, R.M. \& Ghossein, R.A. (2006) Follicular variant of papillary thyroid carcinoma: a clinicopathologic study of a problematic entity. Cancer, 107, 1255-1264.

Liu, Z., Zhou, G., Nakamura, M., Koike, E., Li, Y., Ozaki, T., Mori, I., Taniguchi, E. \& Kakudo, K. (2011) Encapsulated follicular thyroid tumor with equivocal nuclear changes, so-called well-differentiated tumor of uncertain malignant potential: a morphological, immunohistochemical, and molecular appraisal. Cancer Sci., 102, 288-294.

LiVolsi, V.A. \& Baloch, Z. (2020) Noninvasive follicular tumor with papillary-like nuclear features: a practice changer in thyroid pathology. Arch. Pathol. Lab. Med., doi: 10.5858/ arpa.2019-0689-RA. [Epub ahead of print].

Lloyd, R.V., Osamura, R.Y., Klöppel, G. \& Rosai, J. (2017) WHO classification of tumours of endocrine organs, 4th ed., International Agency for Research on Cancer (IARC), Lyon, France.

Maletta, F., Massa, F., Torregrossa, L., Duregon, E., Casadei, G.P., Basolo, F., Tallini, G., Volante, M., Nikiforov, Y.E. \& Papotti, M. (2016) Cytological features of "noninvasive follicular thyroid neoplasm with papillary-like nuclear features" and their correlation with tumor histology. Hum. Pathol., 54, 134-142.

Maletta, F., Volante, M. \& Papotti, M. (2017) Experience on NIFTP cytology, with a mini meta-analysis of the literature. $J$. Basic Clin. Med., 6, 44-50.

Meissner, W. \& Warren, S. (1969) Tumors of the Thyroid Gland 1969 Edition, AFIP, Washington, DC, USA.

Nikiforov, Y.E. (2017) Role of molecular markers in thyroid nodule management: then and now. Endocr. Pract., 23, 979-988.

Nikiforov, Y.E., Baloch, Z.W., Hodak, S.P., Giordano, T.J., Lloyd, R.V., Seethala, R.R. \& Wenig, B.M. (2018) Change in diagnostic criteria for noninvasive follicular thyroid neoplasm with 
papillarylike nuclear features. JAMA Oncol., 4, 1125-1126.

Nikiforov, Y.E., Seethala, R.R., Tallini, G., Baloch, Z.W., Basolo, F., Thompson, L.D., Barletta, J.A., Wenig, B.M., Al Ghuzlan, A., Kakudo, K., Giordano, T.J., Alves, V.A., Khanafshar, E., Asa, S.L., El-Naggar, A.K., et al. (2016) Nomenclature revision for encapsulated follicular variant of papillary thyroid carcinoma: a paradigm shift to reduce overtreatment of indolent tumors. JAMA Oncol., 2, 1023-1029.

Ohba, K., Mitsutake, N., Matsuse, M., Rogounovitch, T., Nishino, N., Oki, Y., Goto, Y. \& Kakudo, K. (2019) Encapsulated papillary thyroid tumor with delicate nuclear changes and a kras mutation as a possible novel subtype of borderline tumor. J. Pathol. Transl. Med., 53, 136-141.

Parente, D.N., Kluijfhout, W.P., Bongers, P.J., Verzijl, R., Devon, K.M., Rotstein, L.E., Goldstein, D.P., Asa, S.L., Mete, O. \& Pasternak, J.D. (2018) Clinical safety of renaming encapsulated follicular variant of papillary thyroid carcinoma: is NIFTP truly benign? World J. Surg., 42, 321-326.

Paulson, V.A., Shivdasani, P., Angell, T.E., Cibas, E.S., Krane, J.F., Lindeman, N.I., Alexander, E.K. \& Barletta, J.A. (2017) Noninvasive follicular thyroid neoplasm with papillary-like nuclear features accounts for more than half of "carcinomas" harboring RAS mutations. Thyroid, 27, 506-511.

Perros, P., Boelaert, K., Colley, S., Evans, C., Evans, R.M., Gerrard Ba, G., Gilbert, J., Harrison, B., Johnson, S.J., Giles, T.E., Moss, L., Lewington, V., Newbold, K., Taylor, J., Thakker, R.V., et al. (2014) Guidelines for the management of thyroid cancer. Clin. Endocrinol. (Oxf.), 81 Suppl 1, 1-122.

Renshaw, A.A. \& Gould, E.W. (2002) Why there is the tendency to "overdiagnose" the follicular variant of papillary thyroid carcinoma. Am. J. Clin. Pathol., 117, 19-21.

Rivera, M., Ricarte-Filho, J., Knauf, J., Shaha, A., Tuttle, M., Fagin, J.A. \& Ghossein, R.A. (2010) Molecular genotyping of papillary thyroid carcinoma follicular variant according to its histological subtypes (encapsulated vs infiltrative) reveals distinct BRAF and RAS mutation patterns. Mod. Pathol., 23, 1191-1200.

Rosario, P.W. (2017) Ultrasonography and cytology as predictors of noninvasive follicular thyroid (NIFTP) neoplasm with papillary-like nuclear features: importance of the differential diagnosis with the invasive encapsulated follicular variant of papillary thyroid cancer. Clin. Endocrinol. (Oxf.), 87, 635-636.

Rosario, P.W. \& Mourão, G.F. (2018) Noninvasive follicular thyroid neoplasm with papillary-like nuclear features (NIFTP) in children and adolescents. Endocrine, 61, 542-544.

Rosario, P.W. \& Mourão, G.F. (2019) Noninvasive follicular thyroid neoplasm with papillary-like nuclear features (NIFTP): a review for clinicians. Endocr. Relat. Cancer, 26, R259R266.

Rosario, P.W., Penna, G.C. \& Calsolari, M.R. (2014) Noninvasive encapsulated follicular variant of papillary thyroid carcinoma: is lobectomy sufficient for tumours $\geq 1 \mathrm{~cm}$ ? Clin. Endocrinol. (Oxf.), 81, 630-632.

Rosario, P.W., Silva, T.H. \& de Oliveira, P.H.L. (2018) Impact of noninvasive follicular thyroid neoplasm with papillary-like nuclear features (NIFTP) on the risk of malignancy estimated by the ultrasonographic classification of the American Thyroid Association (ATA) in thyroid nodules $>1 \mathrm{~cm}$. Endocrine, 60, 535-536.

Rossi, E.D., Mehrotra, S., Kilic, A.I., Toslak, I.E., Lim-Dunham, J., Martini, M., Fadda, G., Lombardi, C.P., Larocca, L.M. \& Barkan, G.A. (2018) Noninvasive follicular thyroid neoplasm with papillary-like nuclear features in the pediatric age group. Cancer Cytopathol., 126, 27-35.

Seethala, R.R., Baloch, Z.W., Barletta, J.A., Khanafshar, E., Mete,
O., Sadow, P.M., LiVolsi, V.A., Nikiforov, Y.E., Tallini, G. \& Thompson, L.D. (2018) Noninvasive follicular thyroid neoplasm with papillary-like nuclear features: a review for pathologists. Mod. Pathol., 31, 39-55.

Seo, J.Y., Park, J.H., Pyo, J.Y., Cha, Y.J., Jung, C.K., Song, D.E., Kwak, J.J., Park, S.Y., Na, H.Y., Kim, J.H., Seok, J.Y., Kim, H.S. \& Hong, S.W. (2019) A multi-institutional study of prevalence and clinicopathologic features of non-invasive follicular thyroid neoplasm with papillary-like nuclear features (NIFTP) in Korea. J. Pathol. Transl. Med., 53, 378-385.

Shafique, K., LiVolsi, V.A., Montone, K. \& Baloch, Z.W. (2018) Papillary thyroid microcarcinoma: reclassification to noninvasive follicular thyroid neoplasm with papillary-like nuclear features (NIFTP): a retrospective clinicopathologic study. Endocr. Pathol., 29, 339-345.

Strickland, K.C., Howitt, B.E., Barletta, J.A., Cibas, E.S. \& Krane, J.F. (2018) Suggesting the cytologic diagnosis of noninvasive follicular thyroid neoplasm with papillary-like nuclear features (NIFTP): a retrospective analysis of atypical and suspicious nodules. Cancer Cytopathol., 126, 86-93.

Volante, M., Collini, P., Nikiforov, Y.E., Sakamoto, A., Kakudo, K., Katoh, R., Lloyd, R.V., LiVolsi, V.A., Papotti, M., SobrinhoSimoes, M., Bussolati, G. \& Rosai, J. (2007) Poorly differentiated thyroid carcinoma: the Turin proposal for the use of uniform diagnostic criteria and an algorithmic diagnostic approach. Am. J. Surg. Pathol., 31, 1256-1264.

Wang, H., Correa, H., Sanders, M., Neblett, W.W. \& Liang, J. (2020) Noninvasive follicular thyroid neoplasm with papillary-like nuclear features in children: an institutional experience and literature review. Pediatr. Dev. Pathol., 23, 121-126.

Williams, E.D. (2000) Guest editorial: two proposals regarding the terminology of thyroid tumors. Int. J. Surg. Pathol., 8, 181-183.

Xu, B., Farhat, N., Barletta, J.A., Hung, Y.P., Biase, D., Casadei, G.P., Onenerk, A.M., Tuttle, R.M., Roman, B.R., Katabi, N., Nosé, V., Sadow, P., Tallini, G., Faquin, W.C. \& Ghossein, R. (2018) Should subcentimeter non-invasive encapsulated, follicular variant of papillary thyroid carcinoma be included in the noninvasive follicular thyroid neoplasm with papillary-like nuclear features category? Endocrine, 59, 143-150.

Xu, B., Reznik, E., Tuttle, R.M., Knauf, J., Fagin, J.A., Katabi, N., Dogan, S., Aleynick, N., Seshan, V., Middha, S., Enepekides, D., Casadei, G.P., Solaroli, E., Tallini, G., Ghossein, R., et al. (2019) Outcome and molecular characteristics of non-invasive encapsulated follicular variant of papillary thyroid carcinoma with oncocytic features. Endocrine, 64, 97-108.

Xu, B., Tallini, G., Scognamiglio, T., Roman, B.R., Tuttle, R.M. \& Ghossein, R.A. (2017) Outcome of large noninvasive follicular thyroid neoplasm with papillary-like nuclear features. Thyroid, 27, 512-517.

Yang, G.C.H., Fried, K.O. \& Scognamiglio, T. (2017) Sonographic and cytologic differences of NIFTP from infiltrative or invasive encapsulated follicular variant of papillary thyroid carcinoma: a review of 179 cases. Diagn. Cytopathol., 45, 533-541.

Yang, G.C.H., Fried, K.O. \& Scognamiglio, T. (2020) Can cytology and the thyroid imaging, reporting, and data system (TI-RADS) identify noninvasive follicular thyroid neoplasm with papillary-like nuclear features (NIFTP) before surgery? $J$. Am. Soc. Cytopathol., 9, 159-165.

Zajkowska, K., Kopczyński, J., Góźdź, S. \& Kowalska, A. (2020) Noninvasive follicular thyroid neoplasm with papillary-like nuclear features: a problematic entity. Endocr. Connect., 9, 47-58. 